\title{
Fuzzy Object retrieval by using histogram of fuzzy Allen relations
}

\author{
Nadeem SALAMAT \\ MIA laboratory \\ University of La Rochelle, Avenue M. Crepeau \\ La Rochelle 17042, France \\ nsalam01@univ-lr.fr
}

\author{
El-hadi ZAHZAH \\ MIA laboratory \\ University of La Rochelle, Avenue M. Crepeau \\ La Rochelle 17042, France \\ ezahzah@univ-lr.fr
}

\begin{abstract}
Relative position of object description are widely used in event understanding and computer vision tasks especially in object recognition. Use of low level features cannot give satisfactory results when high level concepts is not easily expressible in low level contents. Mostly researchers are concentrating on spatio- temporal relationship between objects or regions of an object in images. Object retrieval which is taken into account the relative position of objects in images become important. In such a case classical Allen relations are used. Searched object can take various shapes and scale according to shooting. Fuzzy methods have the ability to compensate the imprecise informations and vagueness.

In this paper fuzzy histograms of Allen relations are used for object retrieval. Fuzzy histograms of Allen relations are the quantitative representation of relative object position. For this purpose Matsakis's [9] algorithm for fuzzification of line segments is refined. This representation is affine invariant. Query is made by example and only corresponding relative relation between objects is considered. Results are analyzed by a well known Receiver Operating Characteristic curve ( ROC )method.
\end{abstract}

Index Terms-Object Retrieval, Fuzzy Allen Relations, Quantitative Representation, Histogram Comparison

\section{INTRODUCTION}

During the last decade, rapid growth in storage capacity of computers and heavy use of interconnected networks have lead to huge amount of numeric data especially images. Indexing systems, allowing users to find images which are relevant to their queries are therefore strongly needed. Content-based image retrieval (CBIR) or query by image content (QBIC) or content-based visual information retrieval (CBVIR) is a technique for retrieving images by apparent object description. These techniques are based on automatically derived features such as color, texture, shape, region within objects, similarity between objects and image ontology [15], [10], [6], [11]. Only a few techniques use high level features such as directional relations [8], [6] and spatio-temporal contents structure [5] but still there is need to improve the systems due to high level semantics. Users need to retrieve images from a collection come from a variety of domains, including medicine, architecture, fashion publishing and crime prevention. It is observed that low level features (contents) cannot describe the high level semantics in the users mind and most of the online image annotation databases are annotated with key words or categories. These key words have direct mapping towards high level semantics which is not one to one [13]. knowledge of relative position of objects in an image or in a scene help a lot in image or scene understanding and recognizing the objects or scene components. Searched object can take various shapes and scale according to shooting and these changes can be described by affine transformations. Regions can be describe by their internal features and relative positions. Relative position representation is robust to shape and scale. Angle histogram [2], and force histograms [3] are real relative position descriptors in literature. These histograms supports a certain topological relations. 1D Allen relations describes the image contents and their relative positions, support each topological relation. These relations are sensitive to shape, size and object orientation.

In this paper, fiat the beginning Matsakis's algorithm [9] for fuzzification of longitudinal section is studied and Allen relations are re-established by $t$-conorms for each segment. Fuzzy Allen relations are used as features and a method for image retrieval is developed. The goal of CBIR is to find all images in a given database that contains user specified visual features, in fuzzy approachs, retrieval does not need to meet the exact decision criteria. In Allen relations intra image grouping of object is taken. This paper is arranged as follows, in next section, used terminology, 1D fuzzy Allen relations and application of $t$-norms and $t$-conorms for coping with segments is explained. In section 3 affine properties of Allen relations are described. In section 4 experiments are explained and methods for histogram comparison, artificial database for experiments and Receiver Operating Characteristic curve for analyzing results are described. Section 5 concludes the paper and in annex-A proof for affine transformations are discussed.

\section{ORIENTED LINES, SEGMENTS AND LONGITUDINAL SECTIONS}

Let $A$ and $B$ are two objects. $(v, \theta) \in R$, where $v$ is any real number and $\theta \in[0,360]$ or $\theta \in[-\pi, \pi] . \Delta_{\theta}(v)$ is an oriented line at orientation angle $\theta . A \cap \Delta_{\theta}(v)$ is the intersection of object $A$ and oriented line $\Delta_{\theta}(v)$. It is denoted by $A_{\theta}(v)$, called segment of object $A$ and its length is $x$. Similarly for object $B$ where $B \cap \Delta_{\theta}(v)=B_{\theta}(v)$ is segment and $z$ is its length. $y$ is the difference between the maximum value of $B \cap \Delta_{\theta}(v)$ and minimum of $A \cap \Delta_{\theta}(v)$ (for details 
see [3]). In case of polygonal object approximations $(x, y, z)$ can be calculated from intersecting points of line and object boundary, oriented lines are considered which passes through at least one vertex of two polygons. If there exist more than one segment then it is called longitudinal section as in case of $A_{\theta}(v)$ in figure 1

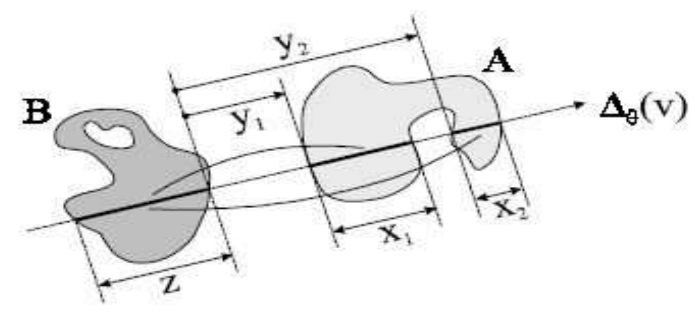

Fig. 1. Oriented line $\Delta_{\theta}(v)$, segment as in case of object $B$, longitudinal section as in case of object A.( Matsakis [3])

\section{A. Allen relations in space}

Interval relations based on temporal interval algebra was introduced by Allen [1]. These relations are arranged as $A=\left\{<, m, o, s, f, d, e q, d_{i}, f_{i}, s_{i}, o_{i}, m_{i},>\right\}$. where $\{<, m, o, s, f, d\}\left(\left\{d_{i}, f_{i}, s_{i}, o_{i}, m_{i},>\right\}\right)$ are the relation before, meet, overlap, start, finish, during (resp the inverse relations of the cited ones). The relation $e q$ is the equality spatial relation. These relations in space are conceptually illustrated in figure 2. These relations have a rich topological support.

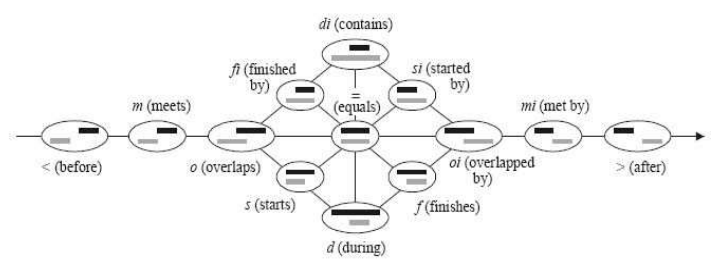

Fig. 2. Black segment represents the reference object and gray segment represents argument object. Figure extracted from [9]

\section{B. Fuzzy Allen relations}

Small errors in crisp values can change the entire result when gradual changes of topological relations occur over time. To cope these problems fuzzification was introduced, it comprises the process of transforming crisp values into grades of fuzzy sets. Fuzzification process of Allen relations do not depend upon particular choice of fuzzy membership function, trapezoidal membership function is used due to its flexibility in shape change. Let $r(I, J)$ is Allen relation between segments $I$ and $J$ where $I \in A$ (argument object) and $J \in B$ (reference object), $d$ is the distance between $r(I, J)$ and its conceptional neighborhood. We consider a fuzzy membership function $\mu: d \longrightarrow[0,1]$. The fuzzy Allen relations defined by Matsakis [9] are

$$
\begin{gathered}
f_{b}(I, J)=\mu_{(-\infty,-\infty,-b-3 a / 2,-b-a)}(y) \\
f_{O}(I, J)=\mu_{(-b-a,-b-a / 2,-b-a / 2, b)}(y) \\
f_{f i}(I, J)=\min \left(\mu_{-b-a / 2,-b,-b,-b+a / 2}(y)\right. \\
, \mu_{(-\infty,-\infty,-(b+a) / 2)}(y) \\
\left., \mu_{(z, 2 z,+\infty,+\infty)}(x)\right) \\
f_{d}(I, J)=\min \left(\mu_{(-b,-b+a / 2,-3 a / 2,-a)}(y)\right. \\
\left., \mu_{(-\infty,-\infty, z / 2, z)}(x)\right)
\end{gathered}
$$

where $a=\min (x, z), b=\max (x, z), x$ is the length of longitudinal section of argument object $A$, and $z$ is the length of longitudinal section of reference object $B$. Most of relations are defined by one membership function and some of them by the minimum value of more than one membership functions like $d$ (during), $d_{i}$ (during_by), $f$ (finish), $f_{i}$ (finished_by). In fuzzy set theory, sum of all the relations is one, this gives the definition for fuzzy relation equal.

\section{Fuzzy operators and treatment of longitudinal sections}

During the decomposition process of an object into segments, there can be multiple segments depending on object shape and boundary which is called longitudinal section. Different segments of a longitudinal section are at a certain distance and these distances might effect end results. Each fuzzy Allen relation is a member of fuzzy set, $T$-normsand $T$ - conorms are used for information integration. Some operators are [12]

- $\mu_{(O R)}(u)=\max \left(\mu_{(A)}(u), \mu_{(B)}(u)\right)$

- $\mu_{(A N D)}(u)=\min \left(\mu_{(A)}(u), \mu_{(B)}(u)\right)$;

- $\mu_{(S U M)}(u)=1-\Pi_{i=1}^{2}\left(\mu_{(i)}(u)\right)$,

- $\mu_{(P R O D)}(u)=\Pi_{i=1}^{2}\left(\mu_{(i)}(u)\right)$

- $\mu_{(\gamma)}(u)=\left[\left(\mu_{(S U M)}(u)\right]^{\gamma} *\left[\left(\mu_{(P R O D)}(u)\right]^{1-\gamma}\right.\right.$ where $\gamma \in$ $[0,1]$

When fuzzy operator $O R$ (respectively $A N D$ ) is used, only one fuzzy value contributes for the resultant value which is maximum (respectively minimum). For other operators both values contribute. In this case each Allen relation has a fuzzy grade and our objective is to accumulate the best available information. In case of longitudinal section, there exist number of segments and each segment has a fuzzy Allen relation with segment of other object. Suppose that longitudinal section of object $A$ has two segments such that $x=x_{1}+x_{2}$ where $x_{1}$ is the length of first segment and $x_{2}$ is the length of second segment and $x$ is length of longitudinal section (as shown in fig. 1). Let $\mu_{1}\left(y_{1}\right)$ defines the value of fuzzy Allen relations with the first segment and $\mu_{2}\left(y_{2}\right)$ represents value of fuzzy Allen relations with the second segment where $y_{1}$ and $y_{2}$ are the distances between object $B$ and two segments of $A$. Now fuzzy $O R$ operator is used to get consequent information obtained from two sets of fuzzy Allen relations $(A N D$ and $\mu_{(P R O D)}(u)$ operators cannot be used). 


\section{Fuzzy histogram of Allen relations}

Histogram of fuzzy Allen relations represents the total area of subregions of $A$ and $B$ that are facing each other in given direction $\theta$ ( [9]). Area of subregions of object $A$ and $B$

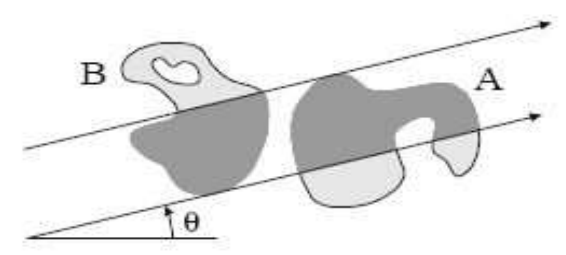

Fig. 3. Histogram of fuzzy Allen relation in direction $\theta$

represented by dark gray color in figure II-D represents a histogram of fuzzy Allen relation in given direction $\theta$. In case of polygonal object approximation, fuzzy histogram of Allen relations can be written as:

$$
\int_{-\infty}^{+\infty}\left(\sum_{r \in A} F_{r}\left(q, A_{q}(v), B_{q}(v)\right) d v=(x+z) \sum_{k=1}^{n} r\left(I_{k}, J_{k}\right)\right.
$$

where $z$ is the area of reference object and $x$ is area of augmented object in direction $\theta, n$ is total number of segments treated and $r\left(I_{k}, J_{k}\right)$ is an Allen relation for segments $I_{k}, J_{k}$. Consider a set of examples. In this example rectangular objects

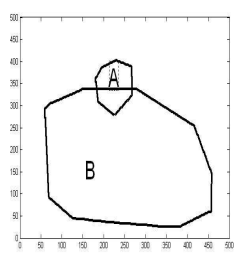

(a)

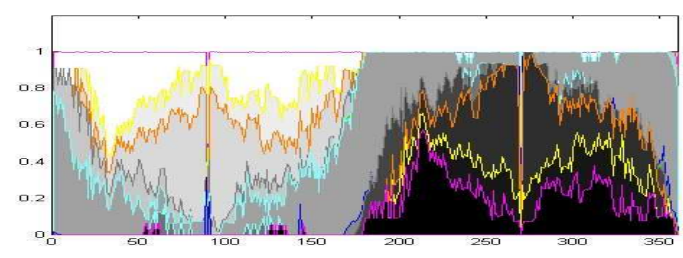

(c)

Fig. 4. (a)Overlapping objects (b)Un normalized fuzzy histogram of Allen relations (c)Corresponding normalized fuzzy histogram of Allen relations

are considered. $A$ firstly for away from the $U$ shaped object $B$. Fuzzy Allen relations are calculated separately for each segment then fuzzy operator is used. Main objective of this example is to show that each segment of longitudinal section has its own impact on Fuzzy Allen relation and each segment may have same, opposite neither opposite nor same Allen relations as in case of fig. 5(a)to fig. 5(e).

In Fig.5(a) object $A$ is at a certain distance to object $B$. Fig.5(b) only after and before relation exists because both

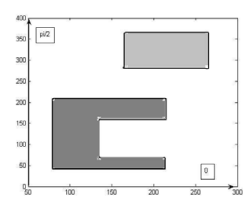

(a)

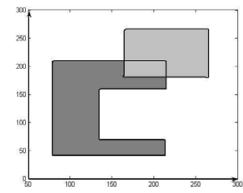

(c)

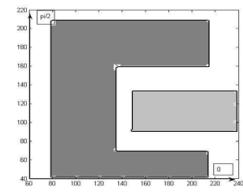

(e)

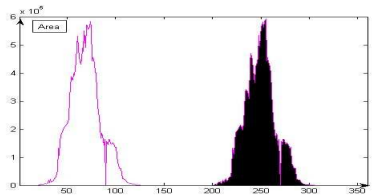

(b)

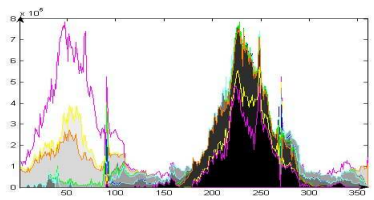

(d)

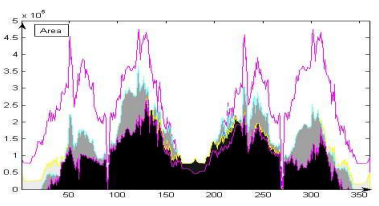

(f)
Fig. 5. Convex and concave pair of objects and their corresponding fuzzy histogram of Allen relations

parts of object $B$ has the same relation. In fig.5(c) when object $A$ is partially overlapped one part of object $B$ then both parts of $B$ has different relations. First part has relations after while second part has has relations like meet_by, overlaped_by along with small value of relation start_by (where meet_by relation due to zigzag). Information for all existing relations for both segments are summarized for a certain angle. Finely in fig.5(e) when object $A$ is between two parts of object $B$, both segments have opposite relations be fore and after meanwhile there exist relation during which is due to zigzag phenomena of digital space and line algorithm.

\section{AFFINE PROPERTIES}

Affinities has their importance in the domain of image recognition especially object matching in a scene analysis. Certain affine properties of fuzzy histograms of Allen relations are depicted below which are independent from fuzzy membership functions.

1) Property: Pair (A, B) is assessable $r$ is any fuzzy Allen relations and for all relations, except the relation during and during_by. $F_{r}^{A, B}(\Theta)=F_{r}^{B, A}(\Theta+\pi)$ And for relation during and during_by we have. $F_{d}^{A, B}(\Theta)=F_{d i}^{B, A}(\Theta)$

2) Property: Let orthogonal symmetry denoted by sym about the oriented line with slop $\alpha$ then histogram of fuzzy Allen relations: $F_{r}^{\text {sym }(A), \operatorname{sym}(B)}(\Theta)=F_{r}^{A, B}(2 \alpha-\Theta)$

3) Property: Let central dilation(scale) denoted by dil and $\lambda$ is dilation ratio then $F_{r}^{\operatorname{dil}(A), \operatorname{dil}(B)}(\Theta)=\lambda^{2} F_{r}^{A, B}(\Theta)$

4) Property: Let stretch (stre) is orthogonal to $\mathrm{x}$ axis and $k$ is stretch ratio, then histogram of fuzzy Allen relations 
satisfies. $F_{r}^{\text {stre }(A), \operatorname{stre}(B)}(\Theta)=\lambda F_{r}^{A, B}(\Theta)$

5) Property: Let translation is denoted by trans and (trans(A), $\operatorname{trans}(\mathrm{B}))$ is assessable then following relation holds for histogram of fuzzy Allen relations. $F_{r}^{\operatorname{trans}(A), \operatorname{trans}(B)}(\Theta)=F_{r}^{A, B}(\Theta)$

6) Property: Let rotation (rot) is a $\rho$-angle rotation and $(\operatorname{rot}(A), \operatorname{rot}(B))$ is assessable hence $F_{r}^{\operatorname{rot}(A), \operatorname{rot}(B)}(\Theta)=F_{r}^{A, B}(\Theta-\rho)$

\section{EXPERIMENTS AND INTERPRETATION}

In this section an image database is built for experimental purpose. Query is composed by the set of regions, adjacent or not adjacent points. Need for retrieving more than one region arises when the semantic object is split into more than one region. A query is composed by characterized spatial relations between the regions or objects within image. Relative object position in image is taken through fuzzy Allen relations. In CBIR or query by example(QBE) query is made by a specific property like "find images where object $A$ is before object $B$ " or by using Allen relations One can say that find images in which object "A (bone) is before object $B$ " (bell), in this way choice of Allen relation is predefined, one can match relation before or after because direction is not mentioned and query resembles the topological relation disjoint.

\section{A. Object pair matching}

Let $(A, B)$ and $\left(A^{\prime}, B^{\prime}\right)$ are two pair of objects. Object pair $(A, B)$ matches $\left(A^{\prime}, B^{\prime}\right)$ through a particular choice of affine transformations such that:

$\left(\mathrm{A}^{\prime}, \mathrm{B}^{\prime}\right)=($ Affine trans $(\mathrm{A})$, Affine $\operatorname{trans}(\mathrm{B}))$

where Affine trans. $=\operatorname{refl}(\operatorname{rot}(\operatorname{stre}(\operatorname{sca}(\operatorname{trans}))))$

and a matching algorithm is described by Matsakis [8].

\section{B. Histogram comparison}

A similarity measure is used to access the degree of similarity of two images according to spatial relations between objects. It is assumed that the images are similar if the distance between corresponding histograms are less than a certain threshold $t$ (Query by example QBE).

$$
\begin{aligned}
M_{6}(A, B) & =\frac{|A \cap B|}{|A \cup B|} \\
& =\frac{\sum_{\theta}[\min (A(\theta), B(\theta))]}{\sum_{\theta}[\max (A(\theta), B(\theta))]} \\
\mu_{T}\left(h_{1}, h_{2}\right) & =\frac{\sum_{\theta}\left(\min \left(h_{1}(\theta), h_{2}(\theta)\right)\right)}{\sum_{\theta}\left(\max \left(h_{1}(\theta), h_{2}(\theta)\right)\right)} \\
\mu_{c}\left(h_{1}, h_{2}\right) & =\frac{\sum_{\theta}\left(h_{1}(\theta) h_{2}(\theta)\right)}{\sqrt{\sum_{\theta}\left(h_{1}^{2}(\theta)\right.} \sqrt{\left.\sum_{\theta} h_{2}^{2}(\theta)\right)}}
\end{aligned}
$$

$M_{6}$ is histogram comparison method discussed in M. Nachtegael et al. [14]. $\mu_{T}$ stands for Tversky index and $\mu_{c}$ is normalized cross correlation for similarity measure. Method for equation 1 can easily be calculated by using $t-n o r m s$ and $t$-conorms. These measures satisfy :
$0 \leq \mu\left(h_{1}, h_{2}\right) \leq 1 ; h_{1}=h_{2} \Longrightarrow \mu\left(h_{1}, h_{2}\right)=1$

$\mu\left(h_{1}, h_{2}\right)=\mu\left(h_{2}, h_{1}\right) ; \mu\left(q h_{1}, q h_{2}\right)=\mu\left(h_{1}, h_{2}\right)$ Histogram measure represented in equation 3 also has the property: $\mu\left(q_{1} h_{1}, q_{2} h_{2}\right)=\mu\left(h_{1}, h_{2}\right)$

\section{Image database}

An artificial data base is created by combining the different test images from $M P E G-7$. Here objects are combined like bell, bone, bottle and brick by changing their positions and size. 30 different views are considered for each object by applying affine transformations. A query is made by using first object in figure 6. For this object only after and before relations exist. Query can be made only using these relations. ROC is drawn to check the efficiency of methods.
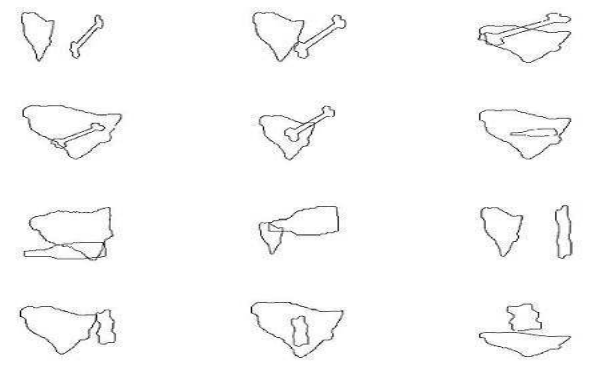

Fig. 6. Object pairs used for data base

\section{ROC curve}

ROC is a well know statistical method to observe the efficiency of a classifier. Let $(A, B)$ and $\left(A_{1}, B_{1}\right)$ are two object pairs, we have to decide either two object pair match or not. In fuzzy techniques it is done with matching degree $\alpha$ output of matching algorithm is greater or equal to some threshold $\tau$. Then there arise four cases.

1) The object pair $(A, B)$ and $\left(A_{1}, B_{1}\right)$ actually match and $\alpha \geq \tau$ : It is a true decision and it is called true positive.

2) The object pair $(A, B)$ and $\left(A_{1}, B_{1}\right)$ actually match and $\alpha<\tau$ : It is a wrong decision and it is called false negative.

3) The object pair $(A, B)$ and $\left(A_{1}, B_{1}\right)$ do not match and $\alpha \geq \tau$ : It is a true decision and we have a false positive.

4) The object pair $(A, B)$ and $\left(A_{1}, B_{1}\right)$ do not match and $\alpha<\tau$ : It is a right decision and it is called true negative.

It is considered that $T P(t)$ be the number of true positive and $F P(t)$ be the number of false positives. Receiver Operating Characteristic (ROC) curve is a plot of the true positive rate against the false positive rate for the different possible cut points of a diagnostic test. In this case its points will be the coordinates of $\left(\frac{T P(\tau)}{93}, \frac{F P(\tau)}{1023}\right)$. Here results are compared by two methods and 31 histograms are associated to each object pair, taking one original and 6 for each transformations (6 by translation, rotation, shear, reflection and scale). Where $t \in$ $[0,1]$ is a threshold. A ROC curve demonstrates several things: 
- It shows the tradeoff between sensitivity and specificity.

- Closer the curve follows the left-hand border and then the top border of the ROC space, more accurate the test.

- Closer the curve comes to the 45-degree diagonal of the ROC space, the less accurate the test.

- The slope of the tangent line at a cut point gives the likelihood ratio (LR) for that value of the test.

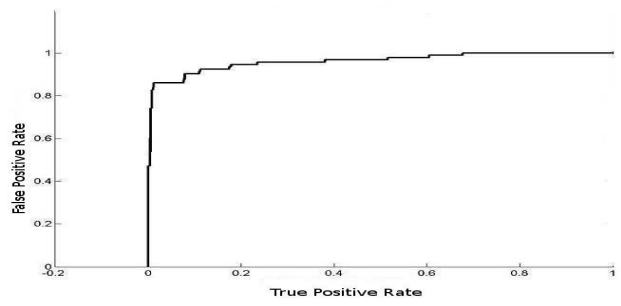

(a)

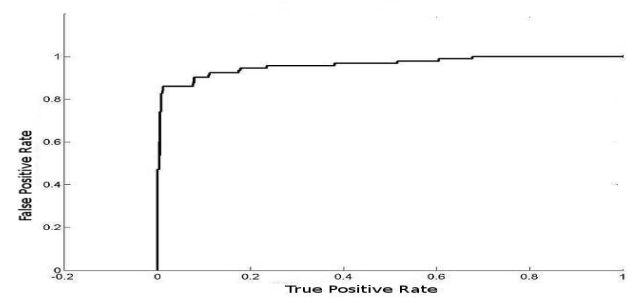

(b)

Fig. 7. (a) ROC for relation before, (b) ROC for relation after

\section{CONCLUSION AND FUTURE WORK}

Fast and fuzzy image searching, browsing and retrieval tools are required due to rapid increase in on line images and data bases. In CBIR images are indexed by visual contents. In this paper first of all Matsakis's work [9] is enhanced. For this purpose fuzzy aggregation operators are used to replace the time consuming fuzzification process for longitudinal sections depicted by Matsakis. This technique is helpful for using quantitative Allen relations in real time applications. At next these fuzzy Allen relations are used for CBIR. These relations are used for object retrieval and relative position of object description, this is a quantitative object description. Allen relations can be used to content based video retrieval (CBVR). Final goal is to find a method which can be successfully used in video data bases. Work will be continue in this field.

\section{REFERENCES}

[1] Allen JF, Maintaining Knowledge about Temporal Intervals , Communications of the ACM 26(11) (1983), 832-843.

[2] A Miyajima, K. Ralescu, Spatial Organization in 2D Images, Fuzzy Systems, 1994. IEEE World Congress on Computational Intelligence, Proceedings of the Third IEEE Conference .

[3] P. Matsakis and L. Wendling. A New Way to Represent the Relative Position between Areal Objects. IEEE Transactions on Pattern Analysis and Machine Intelligence, 21(7):634-643, 1999.

[4] P. Matsakis. Relations Spatiales Structurelles et Interprtation d'Images. $\mathrm{PhD}$ thesis, Institute de Recherche en Informatique de Toulouse,France, 1998.
[5] Dimitris Papadias, Nikos Mamoulis, and Vasilis Delis, Approximate spatio-temporal retrieval, ACM Trans. Inf. Syst. 19 (2001), no. 1, 53-96.

[6] Yuhang Wang and Fillia Makedon, R-histogram: quantitative representation of spatial relations for similarity-based image retrieval, MULTIMEDIA '03: Proceedings of the eleventh ACM international conference on Multimedia (New York, NY, USA), ACM, 2003, pp. 323-326.

[7] Carlos R. P. Dionisio and Hae Yong Kim. New Features for AffineInvariant Shape Classification. In Proceedings of the ICIP 04, International Conference on image Processing, pages 2135- 2138, 2004.

[8] Pascal Matsakis, James M. Keller, Ozy Sjahputera, and Jonathon Marjamaa, The Use of Force Histograms for Affine-Invariant Relative Position Description, IEEE Transactions on Pattern Analysis and Machine Intelligence 26 (2004), no. 1, 1-18.

[9] P.Matsakis, Dennis Nikitenko, Combined Extraction of Directional and Topological Relationship Information from $2 D$ Concave Objects, in fuzzy modeling with spatial informations for geographic problems, SpringerVerlag Publications (M. Cobb, F. Petry, V. Robinson, Eds.), pp. 15-40, New York, 2005

[10] Adel Hafiane, Subhasis Chaudhuri, Guna Seetharaman, and Bertrand Zavidovique, Region-based CBIR in GIS with local space filling curves to spatial representation, Pattern Recognition Letters, 272006, no. 4,259 $-267$.

[11] Agnés Borràs and Josep Lladós, Similarity-based object retrieval using appearance and geometric feature combination, IbPRIA '07: Proceedings of the 3rd Iberian conference on Pattern Recognition and Image Analysis, Part II (Berlin, Heidelberg), Springer-Verlag, 2007, pp. 33-39.

[12] Chang Jo Chung Kwang-Hoon Chi, No-Wook Park, Fuzzy Logic In tergration for Landslide Hazard Mapping using Spatial Data from Boeun,Korea, symposium on geospatial theory,processing and application; ottawa.

[13] Ying Liu, Dengsheng Zhang, Guojun Lu, and Wei-Ying Ma, A Survey of Content-based Image Retrieval with High-level Semantics, Pattern Recognition 40 (2007), no. 1, 262 - 282.

[14] Valerie De Witte1 Tom Mlange1 M.Nachtegael, S.Schulte and Etienne E. Kerre1, Image Similarity From Fuzzy Sets to Color Image Applications, Series Lecture Notes in Computer Science, vol. 4781/2007, pp. 26-37, Springer Berlin / Heidelberg, 2007.

[15] Sylvie Philipp-Foliguet, Julien Gony, and Philippe-Henri Gosselin, FReBIR: An image retrieval system based on fuzzy region matching, Computer Vision and Image Understanding In Press, Corrected Proof (2008).

\section{ANNEX-A}

Histogram of Allen relations is defined as " Area of subregions of object $A$ and $B$ in a particular direction having a relation $r(I, J)$ where $r$ is any Allen relation". Proof of affine transformations can be divided into three parts

1) Effect on change in direction of oriented line.

2) Change in fuzzy membership value

3) Effect of affine transformations on object area

\section{A. Change in direction}

Concerning to the change in direction of oriented line all the proofs are similar as in case of force histogram [4]

\section{B. Change on object area}

It is considered that an object is a polygon, if $A_{k}$ is a transformation matrix of affine transformation $T$ then its effect on polygon $\Delta$ is represented by [7]:

$\operatorname{Area}(T(\Delta))=\left|A_{k}\right| \operatorname{Area}(\Delta)$

Now we calculate effect on area of each transformation and translation is not a affine transformation.

- Scale: Determinant for a matrix of $2 d$ affine transformation for scale is:

$\left|\begin{array}{ll}k & 0 \\ 0 & k\end{array}\right|=k^{2}$

So it will change area of object by $k^{2}$ times. 
- Rotate: Determinant for a matrix of $2 d$ affine transformation for rotate is:

$\left|\begin{array}{cc}\cos (\theta) & -\sin (\theta) \\ \sin (\theta) & \cos (\theta)\end{array}\right|=\cos ^{2}(\theta)+\sin ^{2}(\theta) \Rightarrow 1$

It will not change object area.

- Shear: Determinant for a matrix of $2 d$ affine transformation for shear is:

$\left|\begin{array}{cc}1 & 0 \\ 0 & k\end{array}\right|=k$

So it will change area of object by $k$ times.

- Reflection: Determinant for a matrix of $2 d$ affine transformation for reflection is:

$\left|\begin{array}{cc}\cos (2 \theta) & \sin (2 \theta) \\ \sin (2 \theta) & -\cos (2 \theta)\end{array}\right|=-\left(\cos ^{2}(2 \theta)+\sin ^{2}(2 \theta)\right) \Rightarrow-1$

Its absolute value is 1 so it will not change object area.

\section{Effect on fuzzy membership value}

Grades of fuzzy membership value depends upon triplet $(x, y, z)$. These are the projective distance between two points, so we calculate effect on each point. Let $\left(x_{1}, x_{2}\right)$ be coordinates of a points before transformation and $\left(x_{1}^{\prime}, x_{2}^{\prime}\right)$ are coordinates of a $2 d$ point after transformation.

- Scale: Effect of scale transformation on a projection of a segment :

$$
\begin{aligned}
x_{1}^{\prime} & =\left(\left(\begin{array}{ll}
k & 0 \\
0 & k
\end{array}\right)\left(\begin{array}{l}
x_{1} \\
x_{2}
\end{array}\right)\right)^{t}\left(\begin{array}{l}
1 \\
0
\end{array}\right) \\
& =\left(\begin{array}{l}
k x_{1} \\
k x_{2}
\end{array}\right)^{t}\left(\begin{array}{l}
1 \\
0
\end{array}\right) \\
& =
\end{aligned}
$$

A point after the scale transformation changes $k$ times similarly a segment will also change $k$ times. Now we calculate its effect on a fuzzy membership function. $x^{\prime}=k x$ where $x^{\prime}$ is length of segment after scale transformation and $x$ is length of segment before scale transformation.

$$
\begin{array}{rrr}
\mu_{\left(\alpha^{\prime}, \beta^{\prime}, \gamma^{\prime}, \delta^{\prime}\right)}\left(y^{\prime}\right) & = & \max \left(\min \left(\frac{y^{\prime}-\alpha^{\prime}}{\beta^{\prime}-\alpha^{\prime}}, 1, \frac{\delta^{\prime}-y^{\prime}}{\delta^{\prime}-\gamma^{\prime}}\right), 0\right) \\
& = & \max \left(\min \left(\frac{k y-k \alpha}{k \beta-k \alpha}, 1, \frac{k \delta-k y}{k \delta-k \gamma}\right), 0\right) \\
& = & \max \left(\min \left(\frac{y-\alpha}{\beta-\alpha}, 1, \frac{\delta-y}{\delta-\gamma}\right), 0\right) \\
& = & \mu_{(\alpha, \beta, \gamma, \delta)}(y)
\end{array}
$$

This result shows that scale transformations does not change the value of a fuzzy membership function. This is due to proportional change in value of triplet $(x, y, z)$.

- Rotate: Effect of rotation on a projection of a segment :

$$
\begin{aligned}
x_{1}^{\prime} & =\left(\left(\begin{array}{cc}
\cos (\theta) & -\sin (\theta) \\
\sin (\theta) & \cos (\theta)
\end{array}\right)\left(\begin{array}{l}
x_{1} \\
x_{2}
\end{array}\right)\right)^{t}\left(\begin{array}{l}
1 \\
0
\end{array}\right) \\
& =\left(\begin{array}{c}
\cos (\theta) x_{1}-\sin (\theta) x_{2} \\
\sin (\theta) x_{1}+\cos (\theta) x_{2}
\end{array}\right)^{t}\left(\begin{array}{l}
1 \\
0
\end{array}\right) \\
& =\quad \cos (\theta) x_{1}-\sin (\theta) x_{2}
\end{aligned}
$$

A point after the rotate transformation changes $x^{\prime}=\cos (\theta) x_{1}-\sin (\theta) x_{2}$ which can be rewritten as $x^{\prime}=k_{1} x_{1}-k_{2} x_{2}$

where $\left(x_{1}, x_{2}\right)$ is segment length along $x$ and $y$ axis respectively and $k_{2} x_{2}$ is the projection of $x^{\prime}-x$ on $\mathrm{x}$ axis. This formula (ratio formula in analytic geometry) shows that the length of a segment after the transformation divides the original segment in a particular ratio called $k_{1}, k_{2}$. we can write it as:

$x^{\prime}=\left(k_{1}-k_{2}\right) x=k x$. Where $k=k_{1}-k_{2}$ and $x^{\prime}$ is segment length after transformation and $x$ is segment length before transformation. Now we calculate its effect on a fuzzy membership function.

$$
\begin{aligned}
\mu_{\left(\alpha^{\prime}, \beta^{\prime}, \gamma^{\prime}, \delta^{\prime}\right)}\left(y^{\prime}\right) & = & \max \left(\min \left(\frac{y^{\prime}-\alpha^{\prime}}{\beta^{\prime}-\alpha^{\prime}}, 1, \frac{\delta^{\prime}-y^{\prime}}{\delta^{\prime}-\gamma^{\prime}}\right), 0\right) \\
& = & \max \left(\min \left(\frac{k y-k \alpha}{k \beta-k \alpha}, 1, \frac{k \delta-k y}{k \delta-k \gamma}\right), 0\right) \\
& = & \max \left(\min \left(\frac{y-\alpha}{\beta-\alpha}, 1, \frac{\delta-y}{\delta-\gamma}\right), 0\right) \\
& = & \mu_{(\alpha, \beta, \gamma, \delta)}(y)
\end{aligned}
$$

This proves that rotation does not change the value of a fuzzy membership function. This is due to proportional change in value of triplet $(x, y, z)$.

- Shear: Effect of shear on a projection of a segment :

$$
\begin{aligned}
x_{1}^{\prime} & =\left(\begin{array}{ll}
1 & 0 \\
0 & k
\end{array}\right)\left(\begin{array}{l}
x_{1} \\
x_{2}
\end{array}\right)^{t}\left(\begin{array}{l}
1 \\
0
\end{array}\right) \\
& =\left(\begin{array}{c}
x_{1} \\
k x_{2}
\end{array}\right)^{t}\left(\begin{array}{l}
1 \\
0
\end{array}\right) \\
& =x_{1}
\end{aligned}
$$

A point after the shear transformation does not change. Now we calculate its effect on a fuzzy membership function.

$$
\begin{aligned}
& x^{\prime}=\left(x_{2}^{2}-x_{1}^{1}\right) \Rightarrow x \text { where } x^{\prime} \text { is length of } \\
& \text { segment after shear transformation and } x \text { is } \\
& \text { length of segment before shear transformation. } \\
& \begin{aligned}
\mu_{\left(\alpha^{\prime}, \beta^{\prime}, \gamma^{\prime}, \delta^{\prime}\right)}\left(y^{\prime}\right) & =\max \left(\min \left(\frac{y^{\prime}-\alpha^{\prime}}{\beta^{\prime}-\alpha^{\prime}}, 1, \frac{\delta-y^{\prime}}{\delta^{\prime}-\gamma^{\prime}}\right), 0\right) \\
& =\max \left(\min \left(\frac{y-\alpha}{\beta-\alpha}, 1, \frac{\delta-y}{\delta-k \gamma}\right), 0\right) \\
& =\quad \mu_{(\alpha, \beta, \gamma, \delta)}(y)
\end{aligned} \\
& \text { This completes the proof for shear transformation. }
\end{aligned}
$$

- Reflection: Effect of reflection on a projection of a segment :

$$
\begin{aligned}
x_{1}^{\prime} & =\left(\left(\begin{array}{cc}
\cos (2 \theta) & \sin (2 \theta) \\
\sin (2 \theta) & -\cos (2 \theta)
\end{array}\right)\left(\begin{array}{l}
x_{1} \\
x_{2}
\end{array}\right)\right)^{t}\left(\begin{array}{l}
1 \\
0
\end{array}\right) \\
& =\left(\begin{array}{c}
\cos (2 \theta) x_{1}+\sin (2 \theta) x_{2} \\
\sin (2 \theta) x_{1}-\cos (2 \theta) x_{2}
\end{array}\right)\left(\begin{array}{l}
1 \\
0
\end{array}\right) \\
& =\quad \cos (2 \theta) x_{1}+\sin (2 \theta) x_{2}
\end{aligned}
$$

A point after the reflection transformation changes $x^{\prime}=\cos (2 \theta) x_{1}-\sin (2 \theta) x_{2}$ which can be rewritten as: $x^{\prime}=k_{1} x_{1}-k_{2} x_{2}$ where $\left(x_{1}, x_{2}\right)$ is segment length along $x$ and $y$ axis respectively and $k_{2} x_{2}$ is the projection of $x^{\prime}-x$ on $\mathrm{x}$ axis. This formula ( ratio formula in analytic geometry ) shows that the length of a segment after the transformation divides the original segment in a particular ratio called $k_{1}, k_{2}$. we can write it as: $x^{\prime}=\left(k_{1}-k_{2}\right) x=k x$ Where $k=k_{1}-k_{2}$ and $x$ is segment length before transformation and $x^{\prime}$ is segment length after transformations. Now we calculate its effect on a fuzzy membership function.

$$
\begin{aligned}
\mu_{\left(\alpha^{\prime}, \beta^{\prime}, \gamma^{\prime}, \delta^{\prime}\right)}\left(y^{\prime}\right) & = & \max \left(\min \left(\frac{y^{\prime}-\alpha^{\prime}}{\beta^{\prime}-\alpha^{\prime}}, 1, \frac{\delta^{\prime}-y^{\prime}}{\delta^{\prime}-\gamma^{\prime}}\right), 0\right) \\
& = & \max \left(\min \left(\frac{k y-k \alpha}{k \beta-k \alpha}, 1, \frac{k \delta-k y}{k \delta-k \gamma}\right), 0\right) \\
& = & \max \left(\min \left(\frac{y-\alpha}{\beta-\alpha}, 1, \frac{\delta-y}{\delta-\gamma}\right), 0\right) \\
& = & \mu_{(\alpha, \beta, \gamma, \delta)}(y)
\end{aligned}
$$

This proves that reflection does not change the value of a fuzzy membership function. This is due to proportional change in value of triplet $(x, y, z)$. 